\title{
Breast cancer survival disparity between African American and Caucasian women in Arkansas: A race-by-grade analysis
}

\author{
BEHJATOLAH MONZAVI-KARBASSI ${ }^{1,2}$, ERIC R. SIEGEL ${ }^{3}$, SRIKANTH MEDARAMETLA ${ }^{1}$, \\ ISSAM MAKHOUL $^{2,4}$ and THOMAS KIEBER-EMMONS ${ }^{1,2}$ \\ ${ }^{1}$ Department of Pathology; ${ }^{2}$ Winthrop P. Rockefeller Cancer Institute; ${ }^{3}$ Division of Biostatistics; \\ ${ }^{4}$ Division of Hematology/Oncology, University of Arkansas for Medical Sciences, Little Rock, AR 72205, USA
}

Received August 18, 2015; Accepted May 19, 2016

DOI: $10.3892 / \mathrm{ol} .2016 .4804$

\begin{abstract}
Despite progress in breast cancer treatment, disparity persists in survival time between African American (AA) and Caucasian women in the US. Tumor stage and tumor grade are the major prognostic factors that define tumor aggressiveness and contribute to racial disparity between AA and Caucasian women. Studying the interaction of race with tumor grade or stage may provide further insights into the role of intrinsic biological aggressiveness in disecting the AA-Caucasian survival disparity. Therefore, the current study was performed to evaluate the interaction of race with tumor grade and stage at diagnosis regarding survival in a cohort of patients treated at the Winthrop P. Rockefeller Cancer Institute of the University of Arkansas for Medical Sciences (Little Rock, AR, USA). The cohort included 1,077 patients, 208 (19.3\%) AA and $869(80.7 \%)$ Caucasian, diagnosed with breast cancer between January 1997 and December 2005. Kaplan-Meier survival plots were generated and Cox regressions were performed to analyze the associations of race with breast cancer-specific survival time. Over a mean follow-up time of 1.5 years, AA women displayed increased mortality risk due to breast cancer-specific causes [hazard ratio (HR), 1.74; 95\% confidence interval (CI), 1.23-2.46]. The magnitude of racial disparity varied strongly with tumor grade (race- $\mathrm{X}$-grade interaction; $\mathrm{P}<0.001$ ). No significant interaction was observed between race and tumor stage or race and age at diagnosis. Among women diagnosed with grade I tumors, the race disparity in survival time after controlling for tumor stage and age was strong (HR, 9.07; 95\% CI, 2.11-38.95), but no significant AA-Caucasian disparity was observed among women with higher-grade tumors. The data suggest that, when diagnosed with grade I breast cancer, AA may experience poorer survival outcomes compared with Caucasian patients, regardless of tumor
\end{abstract}

Correspondence to: Dr Behjatolah Monzavi-Karbassi, Department of Pathology, University of Arkansas for Medical Sciences, 4301 West Markham Street, Little Rock, AR 72205, USA

E-mail: karbassi@uams.edu

Key words: breast cancer, health disparity, African American, Caucasian stage or age. The findings potentially provide significant clinical and public health implications and justify further investigation.

\section{Introduction}

An estimated 231,840 novel cases of breast cancer and 40,290 breast cancer-associated mortalities occurred among women from the US in 2015, ranking breast cancer the most common cancer diagnosis and the second leading cause of fatality among American women (1). Although the overall incidence of breast cancer is lower among African American (AA) women compared with Caucasian women, AA breast cancer patients of all ages are more likely to have advanced disease at diagnosis, an increased risk of recurrence and a poorer prognosis (2-6). Racial disparities in breast cancer mortality in the US persist, with AA breast cancer patients experiencing higher mortality rates compared with their Caucasian counterparts (7-9). Recent data from the 50 largest US cities confirms the trend of increasing racial disparities (8). Various factors are proposed to affect this difference in outcome, with an advanced stage at presentation, high-risk tumor biology, lower socioeconomic status, disparity in access to care and treatment and comorbidity typically viewed as the major associated factors $(3,4,10-14)$.

Tumor stage and tumor grade are the major prognostic factors that are commonly used to define the risk of biological aggressiveness of breast tumors $(15,16)$. Tumor stage and grade also contribute to racial disparity between AA and Caucasian women $(17,18)$. It is prudent to understand how race associates with survival in various subgroups defined by tumor stage and grade. In the current study, the disparity in breast cancer mortality among AA and Caucasian women in an Arkansas patient population was investigated. Age, tumor stage and tumor grade were presented for each racial subtype and included in the analysis. The aim of the present study was to examine the interaction of race with tumor grade and with tumor stage, with regards to survival time.

\section{Materials and methods}

Study design and population. A retrospective cohort study was conducted of non-Hispanic AA and Caucasian women, who were treated at the Winthrop P. Rockefeller Cancer Institute of the University of Arkansas for Medical Sciences (UAMS; 
Little Rock, AR, USA). The study was performed according to protocols approved by the UAMS Institutional Review Board (IRB). The UAMS IRB determined the study met the criteria for exempt status per 45 CFR 46, meaning there was no requirement to obtain informed consent from the study subjects. Patients of the two race groups were included if they were 18 years of age or older, diagnosed with histologically confirmed breast cancer between January 1997 and December 2005 and staged into American Joint Committee on Cancer (AJCC) stage groups I, II, III or IV (19). The cohort was followed to assess survival until December 30, 2007. The data were provided by the Arkansas Central Cancer Registry at the Arkansas Department of Health (Little Rock, AR, USA). All women were residents of the Arkansas State. The UAMS Institutional Review Board gave approval for the use of human-subject records in the present study.

Outcome variables. Breast cancer-specific survival, measured as the time to breast cancer-specific fatality, was used as the survival outcome. Survival data were censored at the last contact with the patient when the patient either remained alive or succumbed from causes unrelated to breast cancer. Cause-of-fatality data, classified by International Classification of Diseases (ICD) codes (vol. 9; http://www.cdc.gov/nchs/icd/icd9.htm) were provided by the cancer registry.

Predictor variables. Demographic and clinical variables, taken from the Arkansas tumor registry files, included age at breast cancer diagnosis, date of diagnosis, date of last contact or fatality, race, tumor stage, tumor grade and cause of fatality. The hormone receptor data were not available and therefore not included in the analyses. Breast cancer grade at diagnosis was defined by the AJCC's grade I (well-differentiated), grade II (moderately-differentiated), grade III (poorly-differentiated) or grade IV (undifferentiated). For survival analyses, grades III and IV were combined (grade III/IV). Breast cancer stage at diagnosis used the TNM staging system (20), and included stages 0 , I, II, III, IV and 99. Only patients with known stage were included. Patients with stages '0' (ductal carcinoma in situ) and '99' (unknown) at diagnosis were excluded. Race (including non-Hispanic AAs and Caucasians only), age (as a continuous variable), tumor grade (as a categorical variable) and tumor stage (as a categorical variable) were included as covariates.

Statistical analysis. The $\chi^{2}$ test was used for examining the associations of race with other variables. Survival time was analyzed for covariate associations using univariate and multivariate Cox-regression analyses. Hazard ratios (HRs) and $95 \%$ confidence intervals (CIs) were calculated for each covariate. Two-way interactions between race and the other covariates were evaluated. Survival curves were computed using the Kaplan-Meier method. All P-values were two-sided and evaluated using an $\alpha=0.05$ significance level. IBM SPSS statistics version 22 software (IBM SPSS, Armonk, NY, USA) was used for statistical analyses.

\section{Results}

Characteristics of the studied population. The cohort included a total of 1,077 breast cancer patients, 208 (19.3\%) AA and 869
(80.7\%) Caucasian (Table I). AA women were significantly younger compared with Caucasian women, as close to $1 / 2$ (46.2\%) of AA women were diagnosed at the age of $<50$ years, compared with $<1 / 3(30.6 \%)$ of their Caucasian counterparts. A total of 230 fatalities occurred among the 1,077 breast cancer patients during 1,605 person-years of follow-up; the median follow-up was 0.88 years. Of the total 230 mortalities (21.3\% of the cohort), 152 (66.1\%) patients succumbed due to breast cancer-specific causes. Among AA women, 60 mortalities occurred during 316 person-years of follow-up, 46 (76.7\%) of which were breast cancer-associated. Among Caucasian patients, 170 mortalities occurred during 1,290 person-years of follow-up, $106(62.4 \%)$ of which were attributed to breast cancer.

Breast cancer in the study population were classified into four grades, with grade I exhibiting the fewest and grade IV exhibting the most histological abnormalities. In general, higher-grade tumors are associated with more aggressive behavior, and are considered to have increased relapse and mortality rates $(15,16)$. Tumors categorized as grade III or IV were more common in AA compared with Caucasian women, while grade-I and -II tumors were more common in Caucasian compared with AA women $(\mathrm{P}=0.02)$. More Caucasian patients were diagnosed with stage I tumors compared with AA patients, while more AA compared with Caucasian were diagnosed with stages II, III, and IV ( $\mathrm{P}<0.001)$ (Table I). Distribution of various age groups, tumor stages and grades together with the number of breast cancer-specific deaths are summarized in Table I.

Racial disparity in survival and interaction of race with tumor grade, tumor stage and age. Association of race with breast cancer-specific survival was evaluated. AA women experienced shorter survival (HR, 1.74; 95\% CI, 1.23-2.46; $\mathrm{P}<0.002$ ). Two-way interactions of race with age, race with age groups, race with tumor grade, and race with tumor stage were then analyzed for breast cancer-specific survival (Table II). The only statistically significant interaction observed was between race and tumor grade $(\mathrm{P}<0.001)$. No significant interactions with race were detected for tumor stage $(\mathrm{P}=0.71)$, age $(\mathrm{P}=0.41)$ or age groups $(\mathrm{P}=0.44)$.

Association of race with survival in tumor-grade subgroups. Due to the significant interaction between race and tumor grade and the association with breast cancer-specific survival, survival disparity between Caucasian and AA women was visualized in each tumor-grade subgroup by Kaplan-Meier graphs (Fig. 1). AA showed a dramatic increase in mortality risk compared with Caucasian women when diagnosed with a grade I tumor (Fig. 1A). Since race is associated with tumor grade and stage, and as the survival outcomes are associated with grade, stage and age in addition to race, a multivariate Cox-regression analysis was performed for each tumor-grade subgroup in order to study the effect of race, while adjusting for the other factors (Table III). A statistically significant increase in AA mortality risk was observed even after controlling for age at diagnosis (adjusted HR, 20.08; 95\% CI, 4.95-81.52; P<0.001), tumor stage (adjusted HR, 6.80; 95\% CI, 1.82-25.42; $\mathrm{P}=0.004)$, and age and stage together (adjusted HR, 9.07; 95\% CI, 2.11-38.95; $\mathrm{P}=0.003$ ). When diagnosed with grade II (Fig. 1B) or grade III/IV tumors 
Table I. Characterization of the cohort population used in the present study.

\begin{tabular}{|c|c|c|c|c|}
\hline Characteristic & Caucasian & African American & Total & P-value \\
\hline Race $^{\mathrm{a}}$ & 869 (80.7), 106 & 208 (19.3), 46 & $1,077(100.0), 152$ & NA \\
\hline \multicolumn{5}{|l|}{ Age, years } \\
\hline Min & 27 & 24 & 24 & \\
\hline Max & 96 & 89 & 96 & \\
\hline Mean (SD) & $57.6(13.1)$ & $53.6(14.9)$ & $56.8(13.5)$ & \\
\hline Median & 57 & 52 & 56 & \\
\hline Age group ${ }^{a}$ & & & & $<0.001$ \\
\hline$\leq 50$ & 266 (30.6), 48 & 96 (46.2), 25 & 362 (33.6), 73 & \\
\hline$>50$ & 603 (69.4), 58 & $112(53.8), 21$ & 715 (66.4), 79 & \\
\hline Tumor grade ${ }^{a}$ & & & & 0.020 \\
\hline Grade I & $219(25.2), 6$ & $44(21.2), 8$ & 263 (24.4), 14 & \\
\hline Grade II & 328 (37.7), 29 & $67(32.2), 12$ & 395 (36.7), 41 & \\
\hline Grade III & $304(35.0), 67$ & $87(41.8), 22$ & $391(36.3), 89$ & \\
\hline Grade IV & $18(2.1), 4$ & $10(4.8), 4$ & $28(2.6), 8$ & \\
\hline Tumor stage $^{\mathrm{a}}$ & & & & $<0.001$ \\
\hline Stage I & 360 (41.4), 17 & $56(26.9), 2$ & 416 (38.6), 19 & \\
\hline Stage II & $357(41.1), 48$ & $91(43.8), 18$ & 448 (41.6), 66 & \\
\hline Stage III & 111 (12.8), 28 & 39 (18.8), 14 & 150 (13.9), 42 & \\
\hline Stage IV & $41(4.7), 13$ & 22 (10.6), 12 & $63(5.8), 25$ & \\
\hline
\end{tabular}

${ }^{a}$ Number of patients (\%), number of breast cancer-specific fatalities. NA, not applicable; SD, standard deviation.

Table II. Summary of the results of the interaction analyses between race and other covariates regarding survival endpoint.

\begin{tabular}{lrrr}
\hline Interaction & Wald $\chi^{2}$ & $\mathrm{df}^{\mathrm{a}}$ & P-value \\
\hline Race-X-Grade & 15.83 & 2 & $<0.001$ \\
Race-X-Stage & 1.38 & 3 & 0.710 \\
Race-X-Age & 0.68 & 1 & 0.410 \\
Race-X-Age groups & 0.59 & 1 & 0.440 \\
\hline
\end{tabular}

${ }^{a}$ Degrees of freedom.

(Fig. 1C), no significant race disparity was observed in the presence of age and tumor stage (Table III). Therefore, a substantial and statistically significant increase in mortality risk for patients with lower grade tumors was observed for AA compared with Caucasian breast cancer women.

\section{Discussion}

The present study, in keeping with published data, suggests that AA breast cancer patients exhibit a shorter survival time compared with Caucasian patients $(5,8,20)$. The results are consistent with previously published data from the same institution on data recorded between 1980 and 1998 (21).

While AA women tend to be diagnosed with high-grade tumors, and high tumor grade is considered to contribute to survival disadvantage of AA patients $(18,22)$, the finding of a strong race-by-grade interaction in the present study indicates that tumor grade is an effect modifier for AA-Caucasian disparity over breast cancer-specific survival. This intriguing observation is supported by the subgroup analysis, which suggests that diagnosis at a lower tumor grade, regardless of tumor stage and patient's age at diagnosis, increases AA patients' risk for poor survival. A study reported significant racial disparity among stage I breast cancer patients (23). Tumor grade is considered a determinant for inherent biological differences in tumor aggressiveness that can be controlled by gene expression profile. The data in the present study suggest that AA women experience an additional risk of mortality from breast cancer, but no additional risk compared with Caucasian patients that are already considered high risk due to high tumor grade. The genetic differences between AA and Caucasian women, which remain to be fully explored, may be important in the progression of the cancer and its response to treatment (24-28). Therefore, intrinsic differences in the biology of tumors or the host could be speculated as being involved in observed disparity. Future studies are required to explore such a possibility. Alternatively, it is possible that variations in pharmacogenomics between AA and Caucasian women explain disparity in outcome through differences in responsiveness to chemotherapy or other treatment modalities $(29,30)$. The increased mortality of AA with low-grade tumors may also be due to nonbiological factors like disproportional delays in treatment $(31,32)$, various treatment options $(10,33)$, nonadherence to therapy (34) or health care availability (22). The database used in the present study did not contain this information, and future studies are required to establish the associations between race, tumor grade, treatment response and socioeconomic factors. 
Table III. Hazard ratios for the association between race (African American vs. Caucasian) and breast cancer survival in each tumor grade subgroup.

Hazard ratio (95\% confidence interval) P-value

\begin{tabular}{lccc}
\cline { 2 - 4 } Model & Grade I & Grade II & Grade III/IV \\
\hline Crude & $12.77(3.82-42.66)$ & $1.82(0.92-3.61)$ & $1.13(0.72-1.77)$ \\
& $\mathrm{P}<0.001$ & $\mathrm{P}=0.090$ & $\mathrm{P}=0.600$ \\
Adjusted for age & $20.08(4.95-81.52)$ & $1.61(0.80-3.21)$ & $1.14(0.73-1.79)$ \\
& $\mathrm{P}<0.001$ & $\mathrm{P}=0.180$ & $\mathrm{P}=0.570$ \\
Adjusted for tumor stage & $6.80(1.82-25.42)$ & $1.17(0.56-2.45)$ & $0.98(0.62-1.54)$ \\
& $\mathrm{P}=0.004$ & $\mathrm{P}=0.690$ & $\mathrm{P}=0.920$ \\
Adjusted for age and tumor stage & $9.07(2.11-38.95)$ & $1.00(0.46-2.16)$ & $0.98(0.62-1.56)$ \\
& $\mathrm{P}=0.003$ & $\mathrm{P}=1.000$ & $\mathrm{P}=0.950$ \\
\hline
\end{tabular}

A

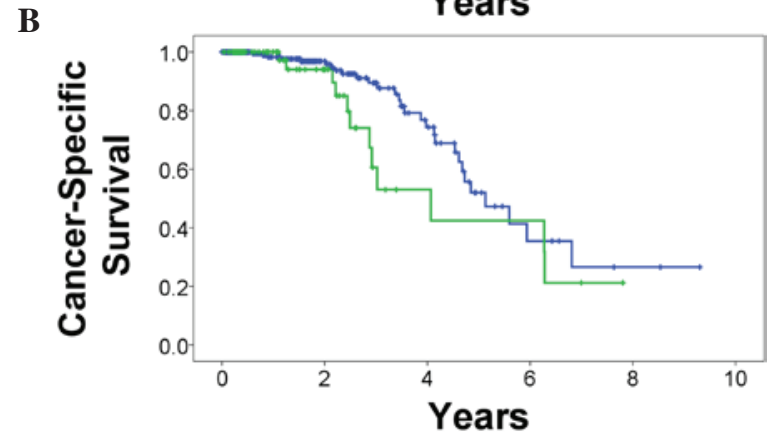

C
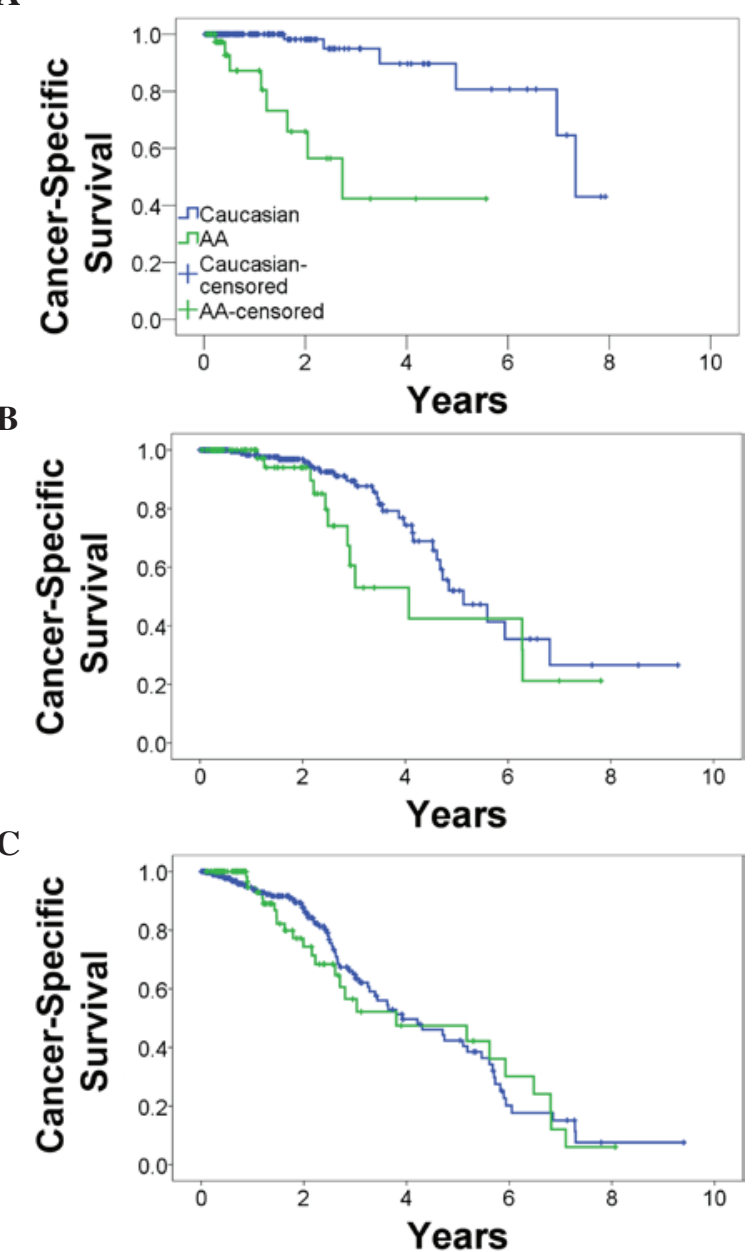

Figure 1. Tumor grade modifies the association of race with breast cancer-specific survival. Kaplan-Meier survival curves for Caucasian vs. AA women diagnosed with (A) grade I, (B) grade II and (C) grade III/IV breast cancer. Please refer to Table III for crude and adjusted hazard ratios and P-values evaluating race disparities in each subgroup. AA, African American.

The increased prevalence of high tumor grade and high tumor stage among AA women and younger age at diagnosis are reported in other studies $(14,17,22,35,36)$. Therefore, the association of race with survival in each grade subgroup may have arisen through confounding with age or tumor stage. However, after controlling for tumor stage and age in the present study, the association of race with cancer-specific survival remained high and statistically significant. This suggests that confounding with age and stage is not sufficient to explain the disparity observed in grade I breast cancer patients between AA and Caucasians. However, due to the relatively low number of cancer-specific fatalities among patients diagnosed with grade I, it is imperative to conduct larger studies on patients with grade I tumors in order to determine whether the present results can be generalized.

Women whose breast cancers are diagnosed as hormone receptor-positive have better survival rates compared with women with receptor-negative tumors, which is most likely associated with the response of tumors to targeted therapies. Diagnosis for triple-negative disease (tumors negative for estrogen receptor, progesterone receptor and human epidermal growth factor receptor 2-neu) is particularly common in young AA patients, who are more likely to suffer a recurrence, be diagnosed with distant metastasis and have a poor prognosis (35,37-39). Despite numerous reports showing that AA breast-cancer women have poorer survival regardless of hormone-receptor status $(40,41)$, there is a possibility that the racial disparity observed in the current cohort could be due to an increased frequency of triple-negative patients in AA women. The hormone-receptor details required to identify basal-like disease were not available for the present study; as a result, the contributions of breast cancer subtypes in the observed racial disparity could not be ruled in or out. Although the possibility that differences in the prevalence of triple-negative cancers contributed to the poorer survival among AA women diagnosed with grade I cannot be excluded in the present study, race disparity is likely to have been even more evident between patients with high grade cancers if triple-negative diagnosis was a contributing factor. Particularly, as a previous study has shown, the increased incidence of basal-like tumors in AA women is associated with higher-grade tumors (42). In other words, the ratio of triple-negative to hormone-receptor positive patients is expected to increase as tumor grade increases. Racial disparity was not observed in grade-II or -III/IV patients; therefore, it is unlikely that the racial disparity observed is due to an increased frequency of triple-negative patients in AA women. 
Another limitation of the present study is the relatively short follow-up time that may potentially affect the outcome of the study. Patients diagnosed with receptor-positive tumors have a relatively better prognosis compared with patients with triple-negative tumors. However this phenomenon is limited to the first 5 years following diagnosis; as time passes, the positive prognostic effect of estrogen receptor status fades away (43). Thus, a short follow-up tends to capture early mortalities compared with a much longer follow-up where the assessment of mortality would be inclusive of early and delayed mortalities. Therefore, the mortality data in the present study could benefit Caucasian over AA women due to a decreased frequency of triple-negative tumors among Caucasian patients. However, as no significant race disparity for grades II and III/IV was observed, the likelihood of a triple-negative diagnosis contributing to the observed racial disparity is diminished. Nevertheless, caution should be exercised when interpreting the results of the present study.

In conclusion, AA breast cancer patients displayed a shorter breast cancer-specific survival time when diagnosed with low-grade tumors. The results of the present study potentially provide significant clinical and public health implications and justify further investigation in larger cohorts.

\section{Acknowledgements}

The authors would like to thank the Winthrop P. Rockefeller Cancer Institute (Little Rock, AR, USA) for supporting the current project.

\section{References}

1. American Cancer Society: Cancer Facts \& Figures 2015. American Cancer Society, Inc., Atlanta, GA, 2015.

2. Ademuyiwa FO and Olopade OI: Racial differences in genetic factors associated with breast cancer. Cancer Metastasis Rev 22: 47-53, 2003.

3. Chlebowski RT, Chen Z, Anderson GL, Rohan T, Aragaki A, Lane D, Dolan NC, Paskett ED, McTiernan A, Hubbell FA, et al: Ethnicity and breast cancer: Factors influencing differences in incidence and outcome. J Natl Cancer Inst 97: 439-448, 2005.

4. Polite BN and Olopade OI: Breast cancer and race: A rising tide does not lift all boats equally. Perspect Biol Med 48 (Suppl 1): S166-S175, 2005.

5. American Cancer Society: Breast Cancer Facts \& Figures 2013-2014. American Cancer Society, Inc., Atlanta, GA, 2013.

6. Keeton KM, Jones ES and Sebastian S: Breast cancer in Mississippi: Impact of race and residential geographical setting on cancer at initial diagnosis. South Med J 107: 510-512, 2014.

7. DeLancey JO, Thun MJ, Jemal A and Ward EM: Recent trends in Black-White disparities in cancer mortality. Cancer Epidemio Biomarkers Prev 17: 2908-2912, 2008.

8. Hunt BR, Whitman S and Hurlbert MS: Increasing black: White disparities in breast cancer mortality in the 50 largest cities in the United States. Cancer Epidemiol 38: 118-123, 2014.

9. McCarthy AM, Yamartino P, Yang J, Bristol M, Conant EF and Armstrong K: Racial differences in false-positive mammogram rates: Results from the ACRIN digital mammographic imaging screening trial (DMIST). Med Care 53: 673-678, 2015.

10. Li CI, Malone KE and Daling JR: Differences in breast cancer stage, treatment, and survival by race and ethnicity. Arch Intern Med 163: 49-56, 2003.

11. Porter PL, Lund MJ, Lin MG, Yuan X, Liff JM, Flagg EW, Coates RJ and Eley JW: Racial differences in the expression of cell cycle-regulatory proteins in breast carcinoma. Cancer 100: 2533-2542, 2004.

12. Amend K, Hicks D and Ambrosone CB: Breast cancer in African-American women: Differences in tumor biology from European-American women. Cancer Res 66: 8327-8330, 2006.
13. Sarker M, Jatoi I and Becher H: Racial differences in breast cancer survival in women under age 60. Breast Cancer Res Treat 106: 135-141, 2007.

14. Baquet CR, Mishra SI,Commiskey P,Ellison GL and DeShields M: Breast cancer epidemiology in blacks and whites: Disparities in incidence, mortality, survival rates and histology. J Natl Med Assoc 100: 480-488, 2008.

15. Rakha EA, Reis-Filho JS, Baehner F, Dabbs DJ, Decker T, Eusebi V, Fox SB, Ichihara S, Jacquemier J, Lakhani SR, et al: Breast cancer prognostic classification in the molecular era: The role of histological grade. Breast Cancer Res 12: 207, 2010.

16. Soerjomataram I, Louwman MW, Ribot JG, Roukema JA and Coebergh JW: An overview of prognostic factors for long-term survivors of breast cancer. Breast Cancer Res Treat 107: 309-330, 2008.

17. Henson DE, Chu KC and Levine PH: Histologic grade, stage and survival in breast carcinoma: Comparison of African American and Caucasian women. Cancer 98: 908-917, 2003.

18. Cunningham JE and Butler WM: Racial disparities in female breast cancer in South Carolina: Clinical evidence for a biological basis. Breast Cancer Res Treat 88: 161-176, 2004.

19. Edge S, Byrd DR, Compton CC, Fritz AG, Greene FL and Trotti A (eds): AJCC Cancer Staging Manual. 7th edition. Springer-Verlag, New York, NY, 2010.

20. Albain KS, Unger JM, Crowley JJ and Coltman CA Jr and Hershman DL: Racial disparities in cancer survival among randomized clinical trials patients of the southwest oncology group. J Natl Cancer Inst 101: 984-992, 2009.

21. Mancino AT, Rubio IT, Henry-Tillman R, Smith LF, Landes R, Spencer HJ, Erkman L and Klimberg VS: Racial differences in breast cancer survival: The effect of residual disease. J Surg Res 100: 161-165, 2001.

22. Danforth DN Jr: Disparities in breast cancer outcomes between Caucasian and African American women: A model for describing the relationship of biological and nonbiological factors. Breast Cancer Res 15: 208, 2013.

23. Iqbal J, Ginsburg O, Rochon PA, Sun P and Narod SA: Differences in breast cancer stage at diagnosis and cancer-specific survival by race and ethnicity in the United States. JAMA 313: 165-173, 2015.

24. Balan V, Nangia-Makker P, Schwartz AG, Jung YS, Tait L, Hogan V, Raz T, Wang Y, Yang ZQ, Wu GS, et al: Racial disparity in breast cancer and functional germ line mutation in galectin-3 (rs4644): A pilot study. Cancer Res 68: 10045-10050, 2008.

25. Field LA, Love B, Deyarmin B, Hooke JA, Shriver CD and Ellsworth RE: Identification of differentially expressed genes in breast tumors from African American compared with Caucasian women. Cancer 118: 1334-1344, 2012.

26. Kalla Singh S, Tan QW, Brito C, De León M and De León D: Insulin-like growth factors I and II receptors in the breast cancer survival disparity among African-American women. Growth Horm IGF Res 20: 245-254, 2010.

27. Stewart PA, Luks J, Roycik MD, Sang QX and Zhang J: Differentially expressed transcripts and dysregulated signaling pathways and networks in African American breast cancer. PLoS One 8: e82460, 2013.

28. Quan L, Gong Z, Yao S, Bandera EV, Zirpoli G, Hwang H, Roberts M, Ciupak G, Davis W, Sucheston L, et al: Cytokine and cytokine receptor genes of the adaptive immune response are differentially associated with breast cancer risk in American women of African and European ancestry. Int J Cancer 134: 1408-1421, 2014.

29. Walko CM, Combest AJ, Spasojevic I, Yu AY, Bhushan S, Hull JH, Hoskins J, Armstrong D, Carey L, Collicio F and Dees EC: The effect of aprepitant and race on the pharmacokinetics of cyclophosphamide in breast cancer patients. Cancer Chemother Pharmacol 69: 1189-1196, 2012.

30. Makhoul I, Klimberg VS, Korourian S, Henry-Tillman RS, Siegel ER, Westbrook KC and Hutchins LF: Combined neoadjuvant chemotherapy with bevacizumab improves pathologic complete response in patients with hormone receptor negative operable or locally advanced breast cancer. Am J Clin Oncol 38: 74-79, 2015.

31. Gwyn K, Bondy ML, Cohen DS, Lund MJ, Liff JM, Flagg EW, Brinton LA, Eley JW and Coates RJ: Racial differences in diagnosis, treatment and clinical delays in a population-based study of patients with newly diagnosed breast carcinoma. Cancer 100: 1595-1604, 2004.

32. George P, Chandwani S, Gabel M, Ambrosone CB, Rhoads G, Bandera EV and Demissie K: Diagnosis and surgical delays in African American and white women with early-stage breast cancer. J Womens Health (Larchmt) 24: 209-2017, 2015. 
33. Griggs JJ, Culakova E, Sorbero ME, Poniewierski MS Wolff DA, Crawford J, Dale DC and Lyman GH: Social and racial differences in selection of breast cancer adjuvant chemotherapy regimens. J Clin Oncol 25: 2522-2527, 2007.

34. Partridge AH, Wang PS, Winer EP and Avorn J: Nonadherence to adjuvant tamoxifen therapy in women with primary breast cancer. J Clin Oncol 21: 602-606, 2003.

35. Morris GJ, Naidu S, Topham AK, Guiles F, Xu Y, McCue P, Schwartz GF, Park PK, Rosenberg AL, Brill K and Mitchell EP: Differences in breast carcinoma characteristics in newly diagnosed African-American and Caucasian patients: A single-institution compilation compared with the national cancer institute's surveillance, epidemiology and end results database. Cancer 110: 876-884, 2007.

36. Barcenas CH, Wells J, Chong D, French J, Looney SW and Samuel TA: Race as an independent risk factor for breast cancer survival: Breast cancer outcomes from the medical college of georgia tumor registry. Clin Breast Cancer 10: 59-63, 2010.

37. Ihemelandu CU, Naab TJ, Mezghebe HM, Makambi KH, Siram SM, Leffall LD Jr, Dewitty RL Jr and Frederick WA: Basal cell-like (triple-negative) breast cancer, a predictor of distant metastasis in African American women. Am J Surg 195: 153-158, 2008.

38. Ihemelandu CU, Leffall LD Jr, Dewitty RL, Naab TJ, Mezghebe HM, Makambi KH, Adams-Campbell L and Frederick WA: Molecular breast cancer subtypes in premenopausal and postmenopausal African-American women: Age-specific prevalence and survival. J Surg Res 143: 109-118, 2007.
39. Lund MJ, Trivers KF, Porter PL, Coates RJ, Leyland-Jones B, Brawley OW, Flagg EW, O'Regan RM, Gabram SG and Eley JW: Race and triple negative threats to breast cancer survival: A population-based study in Atlanta, GA. Breast Cancer Res Treat 113: 357-370, 2009.

40. Menashe I, Anderson WF, Jatoi I and Rosenberg PS: Underlying causes of the black-white racial disparity in breast cancer mortality: A population-based analysis. J Natl Cancer Inst 101: 993-1000, 2009

41. Schinkel JK, Zahm SH, Jatoi I, McGlynn KA, Gallagher C, Schairer C, Shriver CD and Zhu K: Racial/ethnic differences in breast cancer survival by inflammatory status and hormonal receptor status: An analysis of the surveillance, epidemiology and end results data. Cancer Causes Control 25: 959-968, 2014.

42. Carey LA, Perou CM, Livasy CA, Dressler LG, Cowan D, Conway K, Karaca G, Troester MA, Tse CK, Edmiston S, et al: Race, breast cancer subtypes, and survival in the Carolina breast cancer study. JAMA 295: 2492-2502, 2006.

43. Bentzon N, Düring M, Rasmussen BB, Mouridsen H and Kroman N: Prognostic effect of estrogen receptor status across age in primary breast cancer. Int J Cancer 122: 1089-1094, 2008. 